\title{
Nonlinear adjustment of a localized layer of buoyant, uniform potential vorticity fluid against a vertical wall
}

\author{
Karl R. Helfrich* \\ Department of Physical Oceanography, Woods Hole Oceanographic Institution, MS-21, Woods Hole, MA 02543, USA
}

Received 10 October 2005; accepted 8 February 2006

Available online 5 June 2006

\begin{abstract}
The nonlinear evolution of a localized layer of buoyant, uniform potential vorticity fluid with depth $H$, width $w_{0}$ and length $L$ released adjacent to a wall in a rotating system is studied using reduced-gravity shallowwater theory and numerical modeling. In the interior, far from the two ends of the layer, the initial adjustment gives, after ignoring inertia-gravity waves, a geostrophic flow of width $w_{\infty}$ and layer velocities parallel to the wall directed in the downstream direction (defined by Kelvin wave propagation). This steady geostrophic flow serves as the initial condition for a semigeostrophic solution using the method of characteristics. At the downstream end, the theory shows that the fluid intrudes along the wall as rarefaction terminating at a nose of vanishing width and depth. However, in a real fluid the presence of the lower layer leads to a blunt gravity current head. The theory is amended by introducing a gravity current head condition that has a blunt bore joined to the rarefaction by a uniform gravity current. The upstream termination of the initial layer produces a Kelvin rarefaction that propagates downstream, decreasing the layer depth along the wall, and initiating upstream flow adjacent to the wall. The theoretical solution compares favorably to numerical solutions of the reduced-gravity shallow-water equations. The agreement between theory and numerical solutions occurs regardless of whether the numerical runs are initiated with an adjusted geostrophic solution or with the release of a stagnant layer. The latter case excites inertia-gravity waves that, despite their large amplitude and breaking, do not significantly affect the evolution of the geostrophic flow. At times beyond the validity of the semigeostrophic theory, the numerical solutions evolve into a stationary array of vortices. The vortex formation can be interpreted as the finite-amplitude manifestation of a linear instability of the new flow established by the passage of the Kelvin wave. The Kelvin wave ultimately reduces the flux into the downstream gravity current and the vortices retain buoyant in the neighborhood of the initial layer.
\end{abstract} (C) 2006 Elsevier B.V. All rights reserved.

Keywords: Geostrophic adjustment; Gravity currents; Kelvin waves; Nonlinear waves; Vortices

* Tel.: +1 508289 2870; fax: +1 5084572181 .

E-mail address: khelfrich@whoi.edu. 


\section{Introduction}

In the classic geostrophic adjustment problem an initial geostrophically unbalanced state is allowed to relax to a final steady flow whose characteristics are determined by conserving certain properties of the initial state (e.g., mass, potential vorticity and angular momentum) (Rossby, 1938; Blumen, 1972). Often, the adjustment problem is considered for symmetric situations (e.g., the collapse of a cylindrical column) and removed from boundaries. If adjacent to boundary, the initial state is uniform in the direction parallel to the boundary. These restrictions render the adjustment process one dimensional, and in the case against a boundary, eliminate the possibility of Kelvin wave propagation along the boundary.

A notable exception is linear geostrophic adjustment in a channel considered by Gill (1976). In that problem a layer of fluid of depth $h_{1}$ is separated from a layer of the same fluid with depth $H>h_{1}$ by a dam that runs directly across the channel at $x=0$. Removal of the dam excites a Kelvin wave that propagates downstream $(x>0)$ along the right-hand wall (looking from the deep to the shallow layer with anti-clockwise rotation). The Kelvin wave initiates a boundary current that is fed from upstream by another boundary current on the left-hand wall that is established by a second Kelvin wave that propagates back upstream from the dam. The two currents are joined by a cross-channel $(y)$ geostrophic jet at the location of the dam. For an infinitely wide channel this interior flow is just the one-dimensional geostrophic adjustment solution. One of the effects of nonlinearity in the presence of the boundary is the downstream advection of the potential vorticity front established by the fluid depths at $t=0$ (Hermann et al., 1989; Tommason and Melville, 1992; Helfrich et al., 1999). The nose of the potential vorticity front on the right-hand wall moves at a speed that approaches the Kelvin wave speed from below as $h_{1} \rightarrow 0$. When $h_{1}=0$, the downstream Kelvin wave and boundary current are replaced by a rotating gravity current with a blunt bore-like head (Stern et al., 1982; Griffiths and Hopfinger, 1983; Kubokawa and Hanawa, 1984; Helfrich and Mullarney, 2005).

The motion of the potential vorticity front was further analyzed by Stern and Helfrich (2002). They were able to eliminate the complications from the cross-channel jet and a stagnation point on the right wall by taking the initially deeper layer (depth $H$ ) to extend only a finite transverse ( $y$ ) distance from right wall and upstream of $x=0$, and taking the channel width to be infinite. Outside of this deep layer, the ambient fluid again had a depth $h_{1}<H$. The long-time nonlinear evolution of the potential vorticity front intrusion was found using a long-wave, or semigeostrophic, shallowwater theory. After release of the layer, only the right-hand wall Kelvin wave and boundary current remained. The current was fed from upstream by flow parallel to the wall formed by geostrophic adjustment of the transverse step in layer depth. They also used numerical solutions of the shallow-water equations and laboratory experiments to test the theory and explore the effects of a finite-depth lower layer.

The objective is to extend the analysis in Stern and Helfrich (2002) to the case where the depth of the shallow, ambient layer is zero $\left(h_{1}=0\right)$. Of interest is the development of the geostrophically adjusted flow when the initial layer has finite length along the wall. The situation to be considered is depicted in Fig. 1a. A layer of initially motionless, buoyant fluid with density $\rho$, depth $H$, width $w_{0}$ and length $L$ is held adjacent to a vertical wall running in the $x$-direction. The system is rotating about the vertical axis with frequency $f / 2$. The lower layer has density $\rho+\Delta \rho$ and is taken to be deep and motionless.

First consider the infinitely long case $L \rightarrow \infty$. Once released, the layer will spread offshore $(y>0)$ due to gravity until rotation begins to arrest the motion on a timescale $\sim f^{-1}$. Ignoring for the moment high-frequency inertia-gravity waves excited by the release, conservation of volume 
(a)

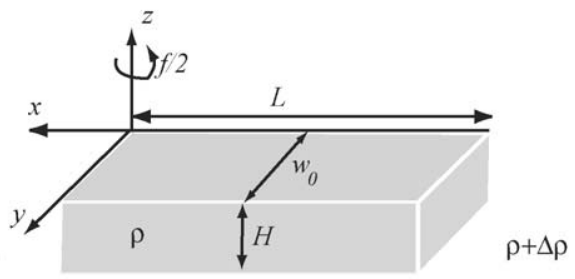

(b)

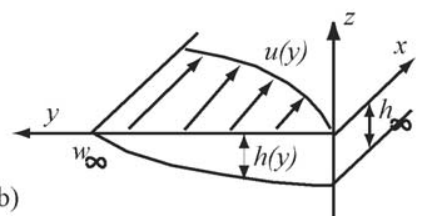

Fig. 1. (a) Sketch of the initial condition. (b) Interior geostrophic flow.

and potential vorticity give the $x$-independent steady, geostrophically adjusted flow

$$
\begin{aligned}
& h(y)=H\left(1-\frac{\cosh \left(y / L_{\mathrm{R}}\right)}{\cosh \left(w_{\infty} / L_{\mathrm{R}}\right)}\right) \\
& u(y)=\left(g^{\prime} H\right)^{1 / 2} \frac{\sinh \left(y / L_{\mathrm{R}}\right)}{\cosh \left(w_{\infty} / L_{\mathrm{R}}\right)} .
\end{aligned}
$$

The width of the adjusted current $w_{\infty}$ is related to the initial width $w_{0}$ by

$$
w_{0}=w_{\infty}-L_{\mathrm{R}} \tanh \left(\frac{w_{\infty}}{L_{\mathrm{R}}}\right),
$$

and

$$
h_{\infty}=H\left[1-\operatorname{sech}\left(\frac{w_{\infty}}{L_{\mathrm{R}}}\right)\right]
$$

is the layer depth at the wall, $y=0$. The deformation radius $L_{\mathrm{R}}=\sqrt{g^{\prime} H} / f, g^{\prime}=g \Delta \rho / \rho$ is the reduced gravity and $g$ is the gravitational acceleration. This adjusted state is shown in Fig. 1b.

When $L$ is finite, there will be additional adjustment processes at both ends of the layer. At the downstream end, $x=0$ (defined by the Kelvin wave propagation direction), the fluid will slump due to gravity and, as in the channel case, form a rotating gravity current that propagates along the wall in the positive $x$-direction. At the upstream end, $x=-L$, initial gravitational slumping will also occur, but it cannot lead to a gravity current moving in the negative $x$-direction since there will be no "right-hand" wall (looking in the negative $x$-direction) to support a geostrophic boundary current. Instead, the upstream adjustment produces a Kelvin wave that propagates in the positive $x$-direction. As will be shown below, disturbances from the downstream end cannot propagate back upstream. Thus, provided $L \gg w_{0}$ these two end adjustment processes will initially proceed independently and the interior geostrophic flow (1)-(2) will remain unchanged until the arrival of the Kelvin wave from the upstream end. The subsequent evolution of the interior and the properties of downstream gravity current are the subjects of this study.

The dynamics of geostrophic adjustment and rotating boundary currents are fundamental geophysical fluid dynamics problems. Possible applications include the relaxation of a downwelling 
ocean front of finite length along the coast after the cessation of the wind and the generation of waves and gravity currents in the marine atmospheric boundary layer along the west coast of the United States (Dorman, 1987). Another example is the relaxation of a pool of dense fluid beneath a coastal polynya after the heat loss has stopped (Chapman and Gawarkiewicz, 1997). These situations are more complicated than the idealized problem outlined above due to the possibility of an active second layer and bottom topography. However, they do share the basic features of the simplified model. Thus, this model should provide some insight into the dynamics and act as a building block for further work.

A reduced-gravity semigeostrophic theory for the evolution at both the downstream and upstream ends is developed in Section 2. In Section 3 the theoretical solutions are compared to numerical solutions of a reduced-gravity shallow-water model including cases where the full adjustment with inertial oscillations is considered. The results are briefly discussed in Section 4 .

\section{Semigeostrophic theory}

A complete theory for the adjustment of the finite-length layer would be very difficult to obtain as the flow is fully nonlinear and time dependent. However, progress can be made if the following assumptions are made. The first, as discussed above, is to consider evolution on times $t>f^{-1}$ after the initial geostrophic adjustment is nearly complete. In the interior, the geostrophic flow in Fig. $1 \mathrm{~b}$ has developed and the initial state in $-L \leq x \leq 0$ is given by (1) and (2). This assumption ignores time-dependent, and possibly dissipative, dynamics that occur as the flow adjusts to the balanced state (Killworth, 1992; Kuo and Polvani, 1997) and also the possible interaction between the inertia-gravity waves and the evolving geostrophic flow.

The role of inertia-gravity waves in geostrophic adjustment has been explored in the weakly nonlinear, small Rossby number, limit by Reznik et al. (2001) and Reznik and Grimshaw (2002). They showed that the initial state can be uniquely split into slow and fast components. The slow, nearly geostrophic, component of the flow is unaffected by the fast inertia-gravity waves on a long timescale proportional to the inverse of the Rossby number. The non-interaction follows from the fact that the inertia-gravity waves do not carry potential vorticity. The Reznik and Grimshaw (2002) study is especially relevant as it considered adjustment adjacent to a wall and found that the presence of a Kelvin wave did not prevent this splitting. This suggests that the assumption to ignore the inertial motions in constructing the solution is reasonable. One caveat is that the problem under consideration has order-one Rossby number and highly nonlinear inertia-gravity waves. This reduces the timescale at which wave-geostrophic flow interaction becomes important.

Additional support for ignoring the inertia-gravity waves comes from the fact that the initial condition has uniform potential vorticity. There are no potential vorticity gradients for the inertia-gravity advect and hence no interaction. This has been discussed by Hayasha and Young (1987) for wave-mean flow interactions in unstable semigeostrophic flows. This implies that the problem here may be a special case. However, Stern and Helfrich (2002) also assumed that the geostrophic adjustment phase could be separated from, and provide the initial condition for, the subsequent nonlinear long-wave evolution in a problem with a potential vorticity front. Some of the inertia-gravity wave energy rapidly radiated away from the adjustment region, though not all. They found comparisons with full numerical solutions of the shallow-water system showed gave good agreement with the semigeostrophic theory.

The second assumption involves the flow at both ends of the initial pool of fluid. Clearly, even the geostrophic flow cannot be valid at either $x=-L$ or 0 , since both ends must undergo independent adjustments. However, any disturbances propagating from the ends move with a 
speed $\approx \sqrt{g^{\prime} H}$, so they can only get a distance $\sim L_{\mathrm{R}}$ on a timescale $f^{-1}$. As long as $L_{\mathrm{R}} / L \ll 1$ the interior solution at $t \sim f^{-1}$ is valid over most of the length of the patch. The last assumption is that the lengthscale of the motion in the $x$-direction, $\lambda$, is long with respect to $L_{\mathrm{R}}$. This long-wave assumption will not be valid for short times in the vicinity of either the upstream or downstream ends, or at the downstream head of the gravity current at later times. Similar application of the long-wave approximation gave excellent results when compared to numerical solutions of the full shallow-water equations for dam-break problems in rotating channels (Helfrich et al., 1999; Stern and Helfrich, 2002; Helfrich and Mullarney, 2005).

There are two non-dimensional numbers that govern the flow. The first is the initial width of the flow divided by the Rossby radius $w_{0} / L_{\mathrm{R}}$ (or $w_{\infty} / L_{\mathrm{R}}$ ) which is the square root of the Burger number $B u$. The second is the aspect ratio $L / w_{0}$. In the analysis below there are no restrictions on $B u^{1 / 2}=w_{0} / L_{\mathrm{R}}$. However, the aspect ratio $L / w_{0} \gg 1$.

With these assumptions, the motion of the active upper layer is governed by the reduced-gravity shallow-water equations (in non-dimensional form)

$$
\begin{aligned}
& \frac{\partial u}{\partial t}+u \frac{\partial u}{\partial x}+v \frac{\partial u}{\partial y}-v=-\frac{\partial h}{\partial x} \\
& \delta^{2}\left(\frac{\partial v}{\partial t}+u \frac{\partial v}{\partial x}+v \frac{\partial v}{\partial y}\right)+u=-\frac{\partial h}{\partial y} \\
& \frac{\partial h}{\partial t}+\frac{\partial}{\partial x}(u h)+\frac{\partial}{\partial y}(v h)=0 .
\end{aligned}
$$

Here $y$ has been non-dimensionalized with $L_{\mathrm{R}}, x$ with $\lambda$, time $t$ with $\lambda / \sqrt{g^{\prime} H}, h$ with $H$ and the velocities $u$ with $\sqrt{g^{\prime} H}$ and $v$ by $\delta \sqrt{g^{\prime} H}$. The parameter $\delta=L_{\mathrm{R}} / \lambda \ll 1$, where $\lambda$ is a lengthscale for the flow in the $x$-direction. The limit $\delta \rightarrow 0$ gives the long-wave, or semigeostrophic, equations (Stern, 1980; Pratt, 1983). The semigeostrophic equations are formally not valid in the times immediately following the release since $\delta=\mathrm{O}(1)$ at both ends, but do become increasingly appropriate as the flow propagates along the wall and $\lambda$ increases (Helfrich et al., 1999; Stern and Helfrich, 2002).

The potential vorticity

$$
q=\frac{1-\partial u / \partial y+\mathrm{O}\left(\delta^{2}\right)}{h},
$$

is conserved following fluid parcels. Substitution of (6), with $\delta=0$, into (8) gives

$$
\frac{\partial^{2} h}{\partial y^{2}}-q h=-1 .
$$

The potential vorticity is scaled with $f / H$ so that $q=1$. The solution to (9), with $h=h_{\mathrm{w}}(x, t)$ at $y=0$ and $h=0$ at $y=w(x, t)$, is

$$
\begin{aligned}
h(x, y, t)= & 1-\frac{h_{\mathrm{w}}(x, t)}{2} \frac{\sinh (y-w(x, t) / 2)}{\sinh (w(x, t) / 2)} \\
& +\left(\frac{1}{2} h_{\mathrm{w}}(x, t)-1\right) \frac{\cosh (y-w(x, t) / 2)}{\cosh (w(x, t) / 2)} .
\end{aligned}
$$


The along-wall velocity

$$
u(x, y, t)=\frac{h_{\mathrm{w}}(x, t)}{2} \frac{\cosh (y-w(x, t) / 2)}{\sinh (w(x, t) / 2)}-\left(\frac{1}{2} h_{\mathrm{w}}(x, t)-1\right) \frac{\sinh (y-w(x, t) / 2)}{\cosh (w(x, t) / 2)},
$$

follows from (6).

The initial geostrophic adjustment gives, from (3) and (4), $w(x, 0)=w_{\infty}$ and $h_{\mathrm{w}}(x, 0)=h_{\infty}$ for $-L<x<0$. The subsequent evolution of $h_{\mathrm{W}}(x, t)$ and $w(x, t)$ can be found by the method of characteristics after reduction of the governing equations to a standard form (Pratt, 1983; Helfrich et al., 1999). This is accomplished by first evaluating the $x$-momentum equation (5) at $y=0$

$$
\frac{\partial u_{\mathrm{w}}}{\partial t}+\frac{\partial}{\partial x}\left(\frac{1}{2} u_{\mathrm{w}}^{2}+h_{\mathrm{w}}\right)=0
$$

and at $y=w(x, t)$

$$
\frac{\partial u_{\mathrm{e}}}{\partial t}-\frac{\partial w}{\partial t}+\frac{\partial}{\partial x}\left(\frac{1}{2} u_{\mathrm{e}}^{2}\right)=0 .
$$

Here $u_{\mathrm{w}}$ and $u_{\mathrm{e}}$ are, respectively, the velocities at the wall and current edge. The kinematic boundary condition at $y=w(x, t)$,

$$
v_{\mathrm{e}}=\frac{\partial w}{\partial t}+u_{\mathrm{e}} \frac{\partial w}{\partial x}
$$

the chain rule

$$
\left.\frac{\partial \phi}{\partial \xi}\right|_{\mathrm{e}}=\frac{\partial \phi_{\mathrm{e}}}{\partial \xi}-\left.\frac{\partial \phi}{\partial y}\right|_{\mathrm{e}} \frac{\partial w}{\partial \xi},
$$

and (6) have been used to obtain (13). With $u_{\mathrm{w}}$ and $u_{\mathrm{e}}$ found from (11), (12) and (13) can be reduced to a $2 \times 2$ quasi-linear system

$$
\frac{\partial \boldsymbol{v}}{\partial t}+\boldsymbol{A} \frac{\partial \boldsymbol{v}}{\partial x}=0
$$

where

$$
\begin{aligned}
& \boldsymbol{v}=\left(\begin{array}{c}
h_{\mathrm{w}} \\
T
\end{array}\right) \\
& \boldsymbol{A}=\left(\begin{array}{cc}
\frac{3 h_{\mathrm{w}}+2 T^{2}+T^{4}\left(h_{\mathrm{w}}-2\right)}{4 T} & \frac{T^{4}\left(h_{\mathrm{w}}-2\right)-(1 / 2) h_{\mathrm{w}}^{2}}{2 T^{2}} \\
\frac{\left(T^{2}-1\right)^{2}\left[h_{\mathrm{w}}-\left(2-h_{\mathrm{w}}\right) T^{2}\right]}{4\left[h_{\mathrm{w}}+\left(2-h_{\mathrm{w}}\right) T^{2}\right]} & \frac{\left(1-T^{2}\right)\left[h_{\mathrm{w}}-\left(2-h_{\mathrm{w}}\right) T^{2}\right]}{4 T}
\end{array}\right),
\end{aligned}
$$

and $T=\tanh (w / 2)$.

The eigenvalues of $\boldsymbol{A}$ are the wave speeds

$$
c_{ \pm}=\frac{1}{2} h_{\mathrm{w}} T^{-1} \pm\left(\frac{1}{2} h_{\mathrm{w}}\right)^{1 / 2}\left[1-\left(1-\frac{1}{2} h_{\mathrm{w}}\right) T^{2}\right]^{1 / 2},
$$




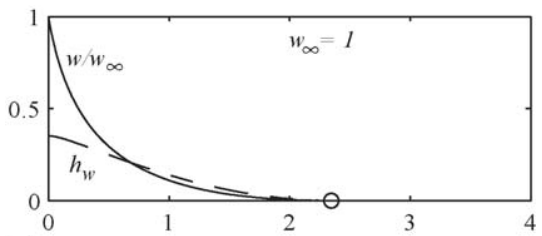

(a)

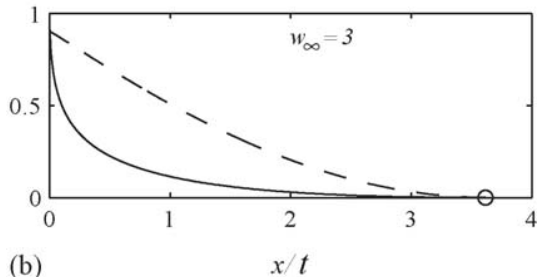

Fig. 2. Downstream rarefaction solution $h_{\mathrm{w}}$ (dashed) and $w / w_{\infty}$ (solid) vs. $x / t$ for (a) $w_{\infty}=1$ and (b) $w_{\infty}=3$. The nose speeds, $c_{\text {nose }}$, are indicated by the circles.

and correspond to a Kelvin wave $\left(c_{+}\right)$and a frontal wave $\left(c_{-}\right)$. When the system (14) is diagonalized using the left eigenvectors of $\boldsymbol{A}$, the relationship

$$
\left.\frac{\mathrm{d} h_{\mathrm{w}}}{\mathrm{d} T}\right|_{ \pm}=\frac{a_{11}-c_{ \pm}}{a_{21}}\left(=\frac{a_{12}}{a_{22}-c_{ \pm}}\right)
$$

must hold on each of the characteristic curves $\mathrm{d} x /\left.\mathrm{d} t\right|_{ \pm}=c_{ \pm}$(Whitham, 1974). Integration of (18) gives the Riemann functions $R_{ \pm}$that are constants along their respective characteristics. Here $a_{i j}$ are the elements of $\boldsymbol{A}$.

\subsection{Downstream end}

The geostrophically adjusted flow with $h_{\mathrm{w}}=h_{\infty}$ and $w=w_{\infty}$ has, from (17), $c_{+}>0$ and $c_{-}=0$. The initial state is critical in the hydraulic sense and long wavelength disturbances from the downstream end cannot propagate back upstream. Thus, until a disturbance from the upstream end reaches $x=0$, the flow there is given by (1) and (2), which will serve as the boundary condition for the downstream flow. The initial uniform flow for $-L<x<0$ implies that one of the Riemann invariants $R_{ \pm}$is the same for all fluid parcels as they flow downstream. It can be shown that taking $R_{-}$uniform gives $u<0$ along the wall $(y=0)$ and $w>w_{\infty}$ when $h_{\mathrm{w}}<h_{\infty}$. This is physically unrealistic since we expect fluid to flow in the positive $x$-direction in a boundary current that decreases in both width and depth downstream of $x=0$. Therefore, $R_{+}$, which does give these properties, is taken to be uniform.

Uniform $R_{+}$gives one relation between $h_{\mathrm{w}}$ and $T$ (or $w$ ) that is found by numerical integration of (18) with $c_{+}$, and the initial condition $T=T_{\infty}=\tanh \left(w_{\infty} / 2\right)$ at $h_{\mathrm{w}}=h_{\infty}$. A second relation between $h_{\mathrm{w}}$ and $T$ comes from consideration of the $R_{-}$Riemann function. Recall that $R_{-}\left(h_{\mathrm{w}}, T\right)$ is constant along $c_{-}\left(h_{\mathrm{w}}, T\right)$ characteristics. Since $T=T\left(h_{\mathrm{w}}\right)$ has already been determined from $R_{+}$, this implies that $R_{-}=R_{-}\left(h_{\mathrm{W}}\right)$ and $c_{-}=c_{-}\left(h_{\mathrm{w}}\right)$. Thus, $R_{-}$is a constant on $c_{-}$only if $h_{\mathrm{w}}$ is constant along the characteristic. Therefore, each $c_{-}$characteristic is constant: $c_{-}\left(h_{\mathrm{W}}, T\left(h_{\mathrm{w}}\right)\right)=x / t$. The solution for $h_{\mathrm{w}}$ and $T$ can be written in terms of a similarity variable $x / t$. 


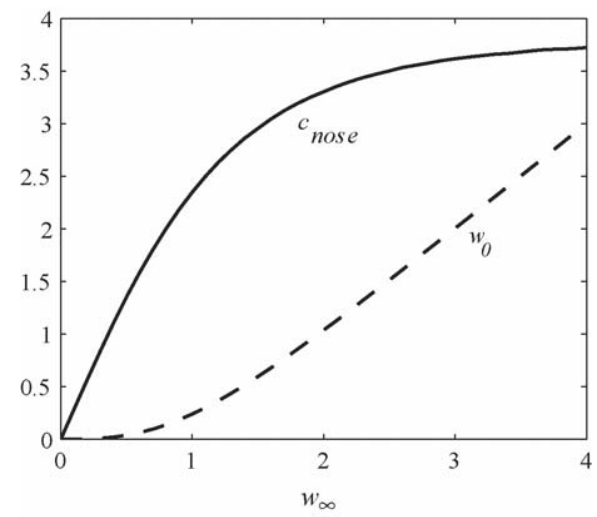

Fig. 3. The downstream rarefaction nose speed $c_{\text {nose }}$ vs. $w_{\infty}$. Also shown is $w_{0}$ (dashed).

Solutions for $w$ and $h_{\mathrm{w}}$ for $w_{\infty}=1$ and 3 are shown in Fig. 2. The fluid flows downstream along the wall as a thinning wedge, or rarefaction, that terminates at a nose with vanishing width and depth. Since both $w$ and $h_{\mathrm{w}}$ vanish at the nose, the speed $c_{\text {nose }}$ is found by applying L'Hospital's rule to (17)

$$
c_{\text {nose }}=\lim _{h_{\mathrm{w}}, T \rightarrow 0} c_{-}=\frac{1}{2} \frac{\mathrm{d} h_{\mathrm{w}}}{\mathrm{d} T} .
$$

To evaluate $c_{\text {nose }}, \mathrm{d} h_{\mathrm{w}} / \mathrm{d} T$ is given by $\mathrm{d} h_{\mathrm{w}} /\left.\mathrm{d} T\right|_{+}$from (18). The numerical evaluation is done for $T=10^{-6}$. The result is not sensitive to smaller values of $T$. The nose speed $c_{\text {nose }}$ is shown in Fig. 3

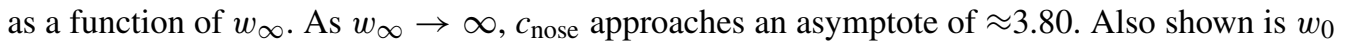
from (3).

\subsubsection{Solutions with a gravity current head}

The rarefying nose solution is equivalent to the classic non-rotating dam-break solution under air in which a rarefying nose moves at the (dimensional) speed $c_{\text {nose }}=2 \sqrt{g H}$ (Stoker, 1957). However, experimental studies demonstrate that these rarefying intrusions are not realized in two-layer systems with small $g^{\prime}$, even if the lower layer is very deep (cf. Huppert and Simpson, 1980). The participation of the lower layer in the momentum and energy budgets of the flow gives a blunt, shock-like, gravity current head, or bore, followed by a nearly uniform current. For an infinitely deep lower layer, the theoretical non-rotating bore speed $c_{\mathrm{b}}=\left(2 g^{\prime} h_{\mathrm{b}}\right)^{1 / 2}$, where $h_{\mathrm{b}}(<H)$ is the height of layer immediately behind the gravity current head (Benjamin, 1968; Klemp et al., 1994, 1997). The situation with rotation is similar, with the thinning rarefaction nose replaced by a blunt rotating gravity current (Stern et al., 1982; Griffiths and Hopfinger, 1983; Kubokawa and Hanawa, 1984; Helfrich and Mullarney, 2005).

Following Abbott (1961) for the non-rotating case, and Helfrich and Mullarney (2005) with rotation, a uniform gravity current will be joined to the rarefaction. Details of the gravity current head condition and the matching for the case of a dam-break in a rotating channel have been discussed in detail in Helfrich and Mullarney (2005). Thus, the analysis will only be outlined. The resulting solutions for the gravity current are qualitatively similar to those obtained in the channel case. The principal difference is the upstream conditions which lead to some quantitative changes. 


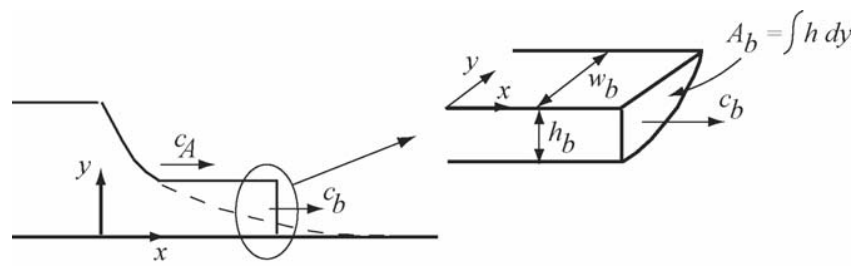

Fig. 4. Schematic of the gravity current. The sketch in the lower-left shows the plan view of the gravity current. The dashed line indicates the rarefaction solution and the solid line shows the blunt gravity current correction. The inset at the right shows a close-up, oblique view of the idealized structure of the gravity current head. The symbols are defined in the text.

The conceptional situation is as depicted in Fig. 4. The gravity current head propagates at a steady speed $c_{\mathrm{b}}$ that, in general, will be a function of the local properties of the gravity current such as $h_{\mathrm{b}}$ and $w_{\mathrm{b}}$, and possibly other parameters such as $f$. Here $w_{\mathrm{b}}$ is the width and $h_{\mathrm{b}}$ is the wall depth of the gravity current. Both are constant from the head back to the junction point at $x_{\mathrm{A}}$, that moves at the constant speed $c_{\mathrm{A}}$.

The first step is to specify the gravity current head conditions. For reduced-gravity semigeostrophic flow Helfrich and Mullarney (2005) showed that a Rankine-Hugoniot solution to the semigeostrophic continuity equation and the $x$-momentum equation evaluated on the wall leads to the (non-dimensional) conditions

$$
\begin{aligned}
& Q_{\mathrm{b}}=A_{\mathrm{b}} c_{\mathrm{b}} \\
& c_{\mathrm{b}}=\frac{1}{2} u_{\mathrm{b}}+\frac{h_{\mathrm{b}}}{u_{\mathrm{b}}} .
\end{aligned}
$$

Here

$$
A_{\mathrm{b}}=\int_{0}^{w_{\mathrm{b}}} h \mathrm{~d} y=w_{\mathrm{b}}+T_{\mathrm{b}}\left(h_{\mathrm{b}}-2\right)
$$

is the cross-sectional area of the gravity current and

$$
Q_{\mathrm{b}}=\int_{0}^{w_{\mathrm{b}}} u h \mathrm{~d} y=\frac{1}{2} h_{\mathrm{b}}^{2}
$$

is the gravity current transport, both evaluated with (10) and (11), and $T_{\mathrm{b}}=\tanh \left(w_{\mathrm{b}} / 2\right)$. The current speed on the wall $u_{\mathrm{b}}$ is found from (11) with $y=0, h_{\mathrm{w}}=h_{\mathrm{b}}$ and $w=w_{\mathrm{b}}$. As with all shock joining solutions, (19) and (20) are integrated closures that do not resolve details of the flow within the gravity current head. They simply guarantee conservation of mass, momentum and energy. Note that energy conservation is a consequence of the infinitely deep lower layer (see Benjamin, 1968; Hacker and Linden, 2002). Fig. 5 shows $c_{\mathrm{b}}$ and $w_{\mathrm{b}}$ from (19) and (20) as functions of $h_{\mathrm{b}}$. Over the range $0<h_{\mathrm{b}}<1, c_{\mathrm{b}} \approx 1.42 h_{\mathrm{b}}^{1 / 2}$, only slightly greater than the non-rotating result $c_{\mathrm{b}}=\sqrt{2 h_{\mathrm{b}}}$. The gravity current width $w_{\mathrm{b}} \approx 0.67 h_{\mathrm{b}}^{1 / 2}$.

An alternative to (20) is the empirical result

$$
c_{\mathrm{b}}=\beta h_{\mathrm{b}}^{1 / 2},
$$

that can be used along with the mass condition (19). Here $\beta \approx 1.2$ from studies of both nonrotating (Huppert and Simpson, 1980) and rotating gravity currents (Stern et al., 1982; Griffiths and Hopfinger, 1983; Kubokawa and Hanawa, 1984; Helfrich and Mullarney, 2005). The reduction 


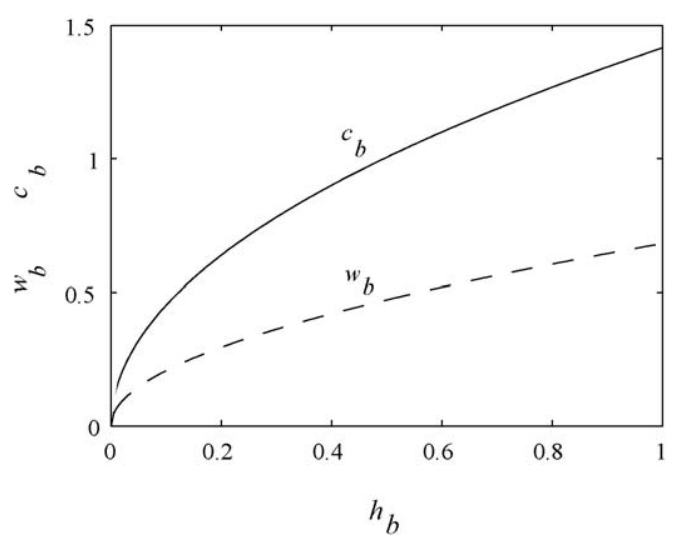

Fig. 5. Gravity current head speed $c_{\mathrm{b}}$ (solid) and width $w_{\mathrm{b}}$ (dashed) vs. wall height $h_{\mathrm{b}}$ from (19) and (20).

in speed from the theoretical model is apparently due to the turbulent mixing and dissipation in the bore head. As a consequence of the slower speed, the gravity current width, $w_{\mathrm{b}} \approx 0.78 h_{\mathrm{b}}^{1 / 2}$, is greater than with (20).

Both of these gravity current head conditions give $c_{\mathrm{b}}$ and $w_{\mathrm{b}}$ as functions of the local value $h_{\mathrm{b}}$. It is necessary to join the gravity current solution to the upstream rarefaction to determine $h_{\mathrm{b}}$ as a function of the upstream conditions. Since $R_{+}$must be uniform, the matching requires that $w_{\mathrm{b}}=w_{\mathrm{b}}\left(h_{\mathrm{b}}\right)$ from the gravity current head solution also satisfy the relation between $w$ and $h_{\mathrm{w}}$ from the rarefaction solution. The simultaneous solution of these two relations gives the bore properties $w_{\mathrm{b}}$ and $h_{\mathrm{b}}$. The speed of the bore $c_{\mathrm{b}}$ and the junction point $c_{\mathrm{A}}=c_{-}\left(h_{\mathrm{b}}, w_{\mathrm{b}}\right)\left(<c_{\mathrm{b}}\right)$ follow. The flow in $0 \leq x \leq x_{\mathrm{A}}$ is given by the rarefaction solution.

Downstream gravity current solutions for $w$ and $h_{\mathrm{w}}$ for $w_{\infty}=1$ and 3 computed with the bore conditions (19) and (20) are shown in Fig. 6. The gravity current properties are summarized in Fig. 7 as functions of $w_{\infty}$ for both choices of head conditions (19) and (20), and (19) and (23)

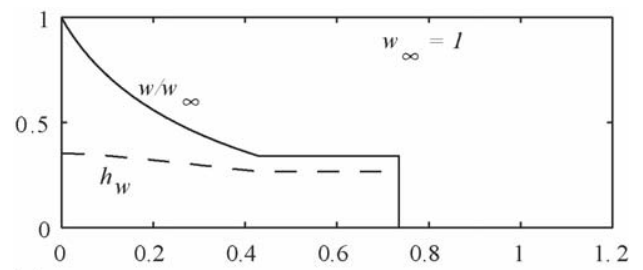

(a)

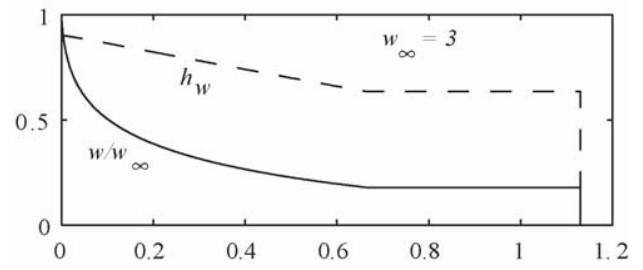

(b)

$x / t$

Fig. 6. Downstream gravity current solutions for $h_{\mathrm{W}}$ (dashed) and $w / w_{\infty}$ (solid) vs. $x / t$ : (a) $w_{\infty}=1$ and (b) $w_{\infty}=3$. 

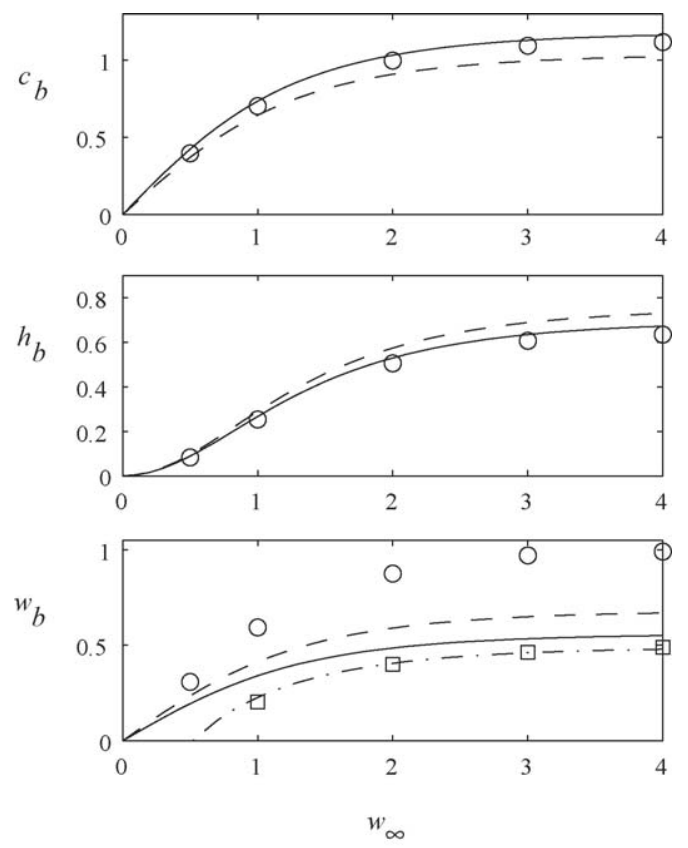

Fig. 7. Gravity current speed $c_{\mathrm{b}}$, height $h_{\mathrm{b}}$ and width $w_{\mathrm{b}}$ as functions of $w_{\infty}$. The semigeostrophic solution with the bore conditions (19) and (20) is shown by the solid lines. The dashed lines are for the bore conditions (19) and (23). The dash-dot line is the offshore width of the gravity current where $h=0.1$ from theory with (19) and (20). The reduced-gravity shallow-water numerical model results for $c_{\mathrm{b}}, w_{\mathrm{b}}$ and $h_{\mathrm{b}}$ are indicated by the circles, and $w$ at $h=0.1$ by the squares.

with $\beta=1.2$. The bore properties $c_{\mathrm{b}}, h_{\mathrm{b}}$ and $w_{\mathrm{b}}$ all increase with $w_{\infty}$. The empirical speed relation (23) with $\beta=1.2$ leads to slower, deeper and wider gravity currents.

\subsection{Upstream end}

The solution at the upstream $(x=-L)$ end is found by again considering that the initial adjustment has produced the basic flow given by $h_{\mathrm{w}}(-L, 0)=h_{\infty}$ and $w(-L, 0)=w_{\infty}$. In this case the solution is a Kelvin wave that propagates downstream. Again, one of the Riemann functions is uniform, and now it is $R_{-}$. Integrating the inverse of (18), with $c_{-}$from (17), from $h_{\mathrm{w}}=h_{\infty}$ to 0 , with the boundary condition $T=T_{\infty}=\tanh \left(w_{\infty} / 2\right)$ at $h_{\mathrm{w}}=h_{\infty}$, gives a relation $T=T\left(h_{\mathrm{W}}\right)$. Since $R_{-}$is uniform, $c_{+}$must be a constant $(=x / t)$ for each $h_{\mathrm{w}}$ in the range $0 \leq h_{\mathrm{w}} \leq h_{\infty}$. The relations between $w, h_{\mathrm{w}}$ and $c_{+}=x / t$ for $w_{\infty}=1$ and 3 are shown in Fig. 8. The solution is a Kelvin rarefaction whose leading edge propagates downstream at speed $c_{\mathrm{K}}=c_{+}\left(h_{\infty}, T_{\infty}\right)$, which increases with $w_{\infty}$ to a maximum of $c_{\mathrm{K}}=1$ for $w_{\infty} \rightarrow \infty$. Behind this leading edge, $h_{\mathrm{w}}$ decreases monotonically to zero at the initial upstream end $x=-L$. The increase of $w$ after passage of the wave is greatest for narrow currents, consistent with a Kelvin wave trapped to the wall.

Since $c_{-}<0$ for $-L<x<-L+c_{\mathrm{K}} t$, the interaction of the Kelvin wave with the transition to the downstream solution at $x=0$ will generate a reflected frontal wave that moves back upstream. Thus, this upstream solution is valid until $t=L / c_{\mathrm{K}}$. The arrival of the Kelvin wave at $x=0$ will also initiate a decrease in the flux into the downstream gravity current. 

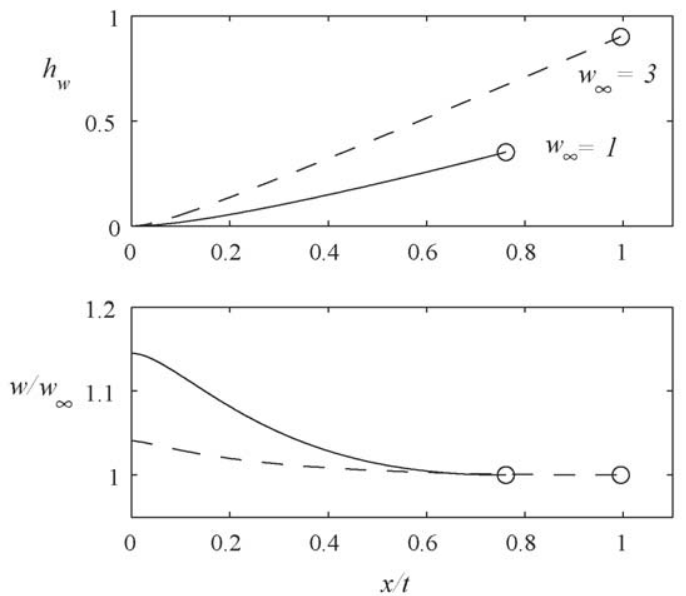

Fig. 8. Upstream Kelvin rarefaction solutions for $h_{\mathrm{w}}$ and $w / w_{\infty}$ vs. $x / t$ for $w_{\infty}=1$ (solid) and $w_{\infty}=3$ (dashed). The circles indicate the leading edge of the wave.

An example of the complete semigeostrophic solution for $w_{\infty}=2$ and $L=30$ is shown in Fig. 9. In this and subsequent figures the lengthscale $\lambda$ has be set to $L_{\mathrm{R}}$. Contours of the layer depth $h(x, y, t)$ are shown at $t=0$ (the initial geostrophically adjusted state), 12.5 and 25. At a fixed $x$ in $-L \leq x \leq 0$, the passage of the Kelvin rarefaction causes the depth on the wall to decrease with time. This, in turn, causes the fluid adjacent to the wall to flow
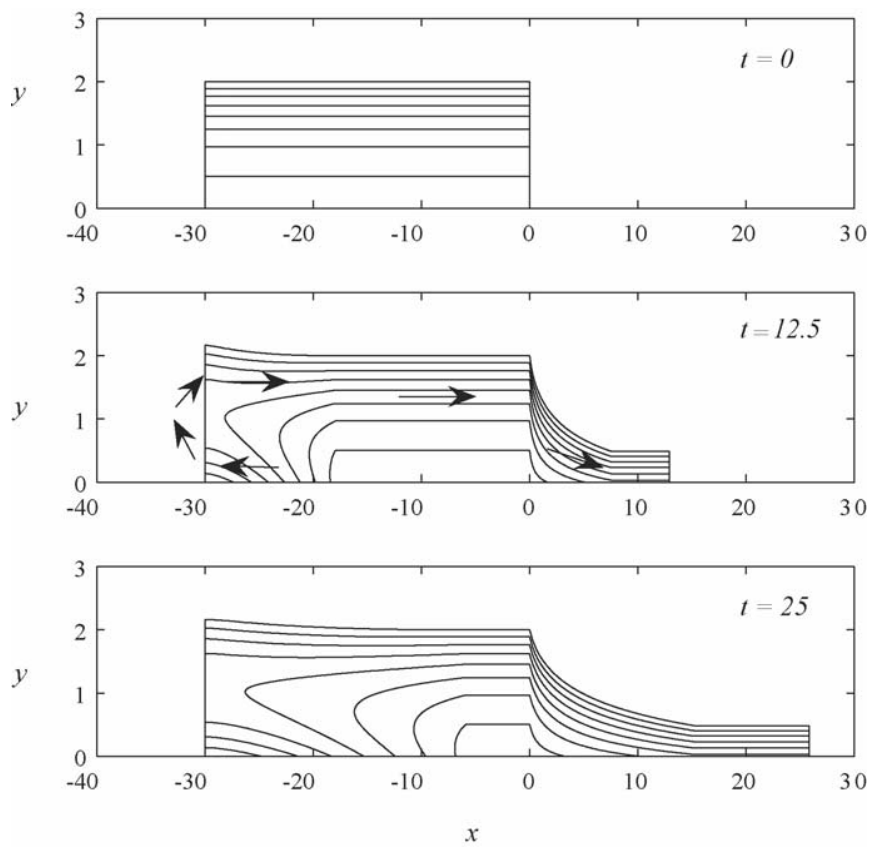

Fig. 9. Complete semigeostrophic solution for $w_{\infty}=2$ and $L=30$. Contours of $h(x, y, t)$ are shown at $t=0,12.5$ and 25 . The contour interval is 0.1 . The arrows indicate the flow direction. 
back upstream, creating a bidirectional flow. At $x=-L, h_{\mathrm{w}}=0$ and thus the net geostrophic transport in the current is zero. However, there is upstream flow adjacent to the wall, and downstream flow in the offshore half of the current. The semigeostrophic solution does not address how the upstream flow turns to join the offshore downstream flow, or if it even can. This transition must occur in an area with $\delta=\mathrm{O}(1)$ where the long-wave approximation is not valid.

\section{Shallow-water numerical modeling}

The theoretical model hinges on two significant assumptions. The first is that $\delta \ll 1$, a condition that is violated by the initial condition. The second is that the fast inertia-gravity waves excited in the initial release can be ignored. These assumptions will be tested by comparing the theoretical solutions to those obtained from a numerical solution of the full shallow-water equations. The numerical modeling will also explore the evolution for $t>L / c_{\mathrm{K}}$.

One requirement of the model is the ability to develop blunt bores. When the momentum equations are written in flux form (i.e., with dependent variables $U=u h$ and $V=v h$ ) the shallowwater equations do not admit a blunt bore-like gravity current because the Rankine-Hugoniot shock solution gives $c_{\mathrm{b}} \rightarrow \infty$ for $u=h=0$ ahead of the bore. Thus, the numerical model solves the shallow-water equations in the advective form, (5)-(7) (with $\delta=1$ ), which naturally develop a blunt gravity current head. Both mass and momentum will be conserved across a bore (Benjamin, 1968). If a locally steady bore is observed in a frame moving at the bore speed, the advective form of the equations will conserve the Bernoulli function across the bore. However, when viewed in the fixed frame, energy will be lost since the flow is unsteady. The Bernoulli function is no longer a conserved quantity. Thus, the bore, and any other wave breaking that might occur in the calculations, result in a loss of energy from the system. Potential vorticity is conserved unless the breaking induces non-uniform energy loss along the breaking face. Fluid parcels that pass through the breaking wave then undergo a change in potential vorticity (Pratt, 1983). One deficiency of the advective form of the equations is that should the flow develop a shock joining regions of finite depth, the shock properties will not be modeled correctly. Momentum is not a conserved quantity.

The numerical solution technique is derived from the second-order finite-volume method for the conservative form of the single-layer shallow-water equations described in Helfrich et al. (1999). The only modification necessary for the present problem is a change in the computation of the nonlinear terms in the momentum equations from a conservative formulation to the advective form of (5) and (6). These are handled using the scalar advection (or 'color') scheme described in Leveque (2002, Sections 9.3 and 20.5). The flux form of the continuity equations requires the related conservative advection scheme (Section 9.5.2). The pressure gradient and Coriolis terms are handled via Strang splitting. The model is exactly mass conserving, but will not conserve energy or potential vorticity in the situations just described. The shallow-water model has been successfully tested in a number of rotating flow problems involving shocks, hydraulic jumps, the presence of zero layer depths and gravity currents (e.g., Pratt et al., 2000; Helfrich and Mullarney, 2005).

The runs were conducted in rectangular channels of various lengths in the $x$-direction and widths large enough to avoid flow interaction with the offshore boundary. A no-flux boundary condition was employed at $y=0$ and radiation boundary conditions in $x$. The calculations had grid spacing $\mathrm{d} x=0.1$ and $\mathrm{d} y=0.0125-0.05$. The layer is considered to have zero thickness where $h \leq 10^{-3}$. The results are insensitive to small values for the cutoff. No explicit diffusion or friction 
(a)

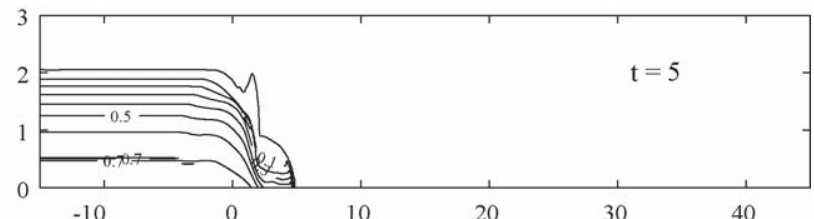

)

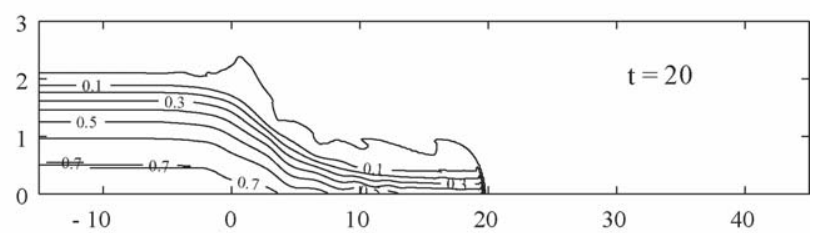

(b)

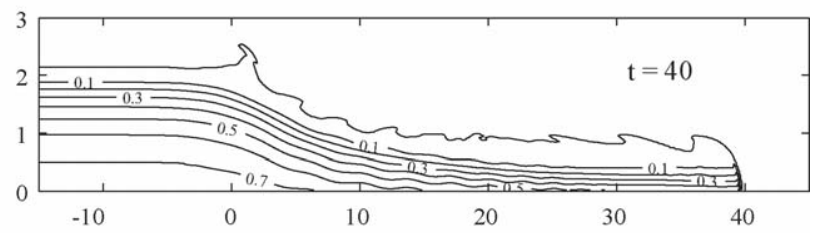

(c)

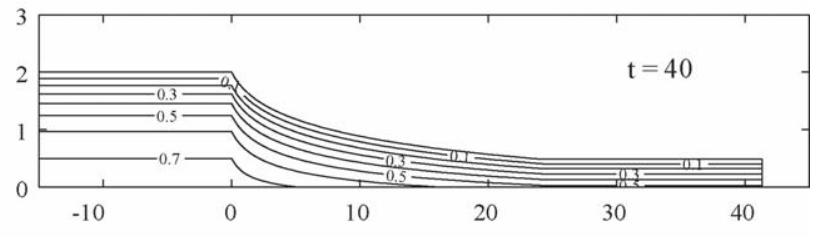

(d)

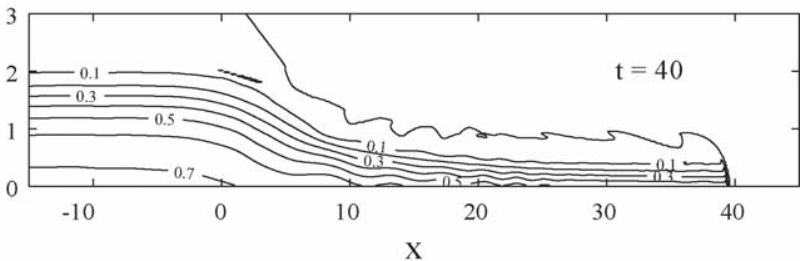

Fig. 10. Solutions for the downstream gravity current for $w_{\infty}=2$. (a-c) The reduced-gravity numerical solution computed with the adjusted initial condition at $t=5,20$ and 40. (d) The semigeostrophic solution at $t=40$. (e) Numerical solution at $t=40$ computed with the still initial condition $w_{0}=1.03$. All panels show contours of $h(x, y, t)$.

terms were included in the present calculations. There is, of course, some numerical dissipation, but it is relatively unimportant except in vicinity of a discontinuity.

\subsection{Downstream gravity current}

The downstream gravity current evolution is explored first in a series of numerical runs with the initial condition given, as in the semigeostrophic theory, by the adjusted geostrophic flow (1) and (2) for $x \leq 0$. The solution for $w_{\infty}=2$ at $t=5,20$ and 40 is shown in Fig. 10a-c. The corresponding semigeostrophic characteristic solution at $t=40$ is shown in Fig. 10d. The overall 
agreement between the numerical solution and the theory is quite good. Both the rarefaction and uniform gravity current emerge in the numerical solution. The gravity current head propagates at a constant speed $c_{\mathrm{b}}=1.0$, slightly slower than the theoretical prediction $c_{\mathrm{b}}=1.03$. The height of the gravity current on the wall just behind the head is $h_{\mathrm{b}}=0.51$ and the predicted height is 0.529 .

The largest difference between the theory and the numerical solution is the width of the gravity current. In the numerical solution the gravity current width is not uniform and is significantly wider than predicted. The average width $w_{\mathrm{b}}=0.88$, compared to $w_{\mathrm{b}}=0.486$ from the theory. This discrepancy is due to details of the ageostrophic flow in the numerically computed gravity current head and has been discussed in Helfrich and Mullarney (2005) (see their Fig. 8). In summary, in the reference frame traveling with the gravity current head, fluid adjacent to the wall flows towards the head to form an offshore jet in a narrow boundary layer just behind the leading edge of the gravity current. The jet squirts fluid offshore to produce the shallow and wide layer with upstream flow relative to $c_{\mathrm{b}}$. Recall that the semigeostrophic gravity current model, like all shock-joining theories, does not resolve the details of the flow within the gravity current head and so cannot capture this effect. The numerically determined flow is qualitatively similar to some rotating gravity currents observed in the laboratory (Stern et al., 1982; Griffiths and Hopfinger, 1983; Kubokawa and Hanawa, 1984) and to the ageostrophic boundary layer jet that connects a Kelvin shock to a trailing geostrophic flow (Fedorov and Melville, 1996; Pratt et al., 2000).

The numerical results for the gravity current properties $c_{\mathrm{b}}, h_{\mathrm{b}}$ and $w_{\mathrm{b}}$ are summarized in Fig. 7. The overall agreement between the theory and numerical model for $c_{\mathrm{b}}$ and $h_{\mathrm{b}}$ is very good. As explained above, the numerical results for $w_{\mathrm{b}}$ are larger than the theoretical values. However, the width of the gravity current at the point where $h=0.1$ agrees with the theory.

If the initial condition is the dammed region of width $w_{0}$ (Fig. 1a), instead of the geostrophically adjusted state, the gravity current properties are essentially unchanged. This is illustrated in Fig. 10e where the solution at $t=40$ for a run with $w_{0}=1.03\left(w_{\infty}=1.994\right)$ is shown. In this case, $c_{\mathrm{b}}=0.98, h_{\mathrm{b}}=0.49$ and the average $w_{\mathrm{b}}=0.87$ are all within a few percent of the values found with the geostrophically adjusted initial condition. This agreement extends to other initial values of $w_{0} \leq 3\left(w_{\infty} \leq 4\right)$. The only substantial differences between Fig. 10c and e are in the region $x<5$. Overshoot and inertial oscillations produced by the initial gravitational slumping lead to a very thin offshore layer of depth $h \approx 0.01$ extending from $y \approx 2.2$ to 3.4.

\subsection{Upstream flow}

The numerical solution for a case with a finite upstream length $L=30$ is shown in Fig. 11 . The run was made with the geostrophic solution (1) and (2) with $w_{\infty}=2$ as the initial condition in $-L \leq x \leq 0$. At $t=20$ the solution is in qualitative agreement with the semigeostrophic theory (Fig. 9). The Kelvin rarefaction has decreased the depth along the wall and initiated an upstream flow. At the upstream end, the fluid turns to rejoin the offshore flow back towards the gravity current. However, the current width at the upstream end is wider than predicted and exhibits damped oscillations in $x$.

Fig. 12 shows a companion run, but now initiated with the stagnant pool. Here $w_{0}=1.03$ $\left(w_{\infty}=1.994\right)$ and $L=30$. The differences between this solution and Fig. 11b are minor. All the important qualitative features are present.

On closer inspection there are differences between the upstream solutions with the two initial conditions as highlighted in Fig. 13. The temporal evolution layer depth at the wall $h_{\mathrm{w}}(x, t)$ at $x=-22.5$ is shown in Fig. 13a. The profile of $h_{\mathrm{w}}$ versus $x$ at several times, and the offshore structure of the layer depth at $x=-15$ are given in Fig. $13 \mathrm{~b}$ and c, respectively. First, in all three 


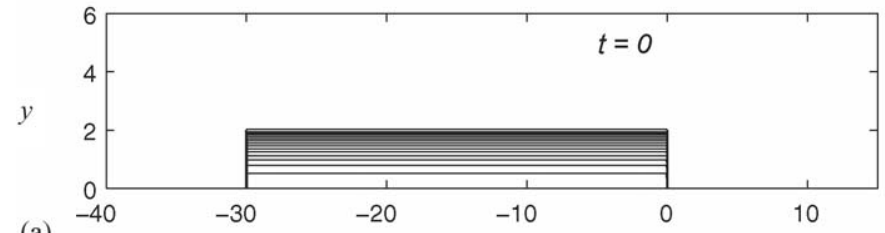

(a)

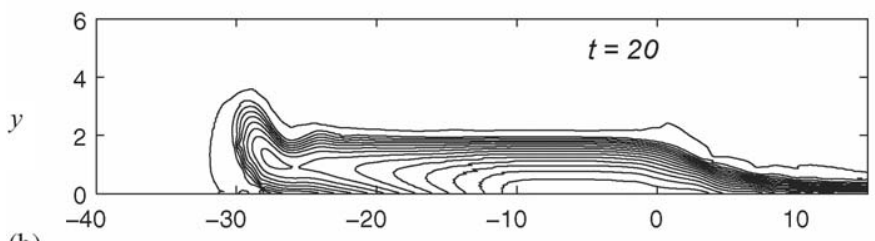

(b)

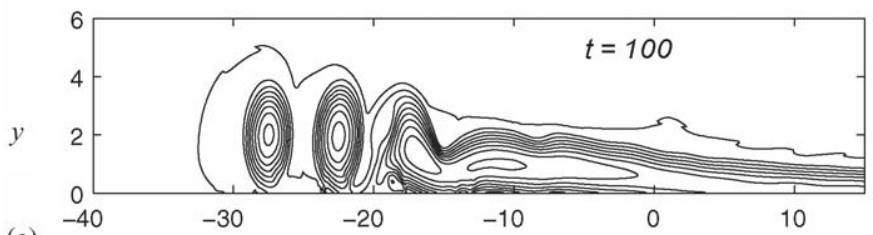

(c)

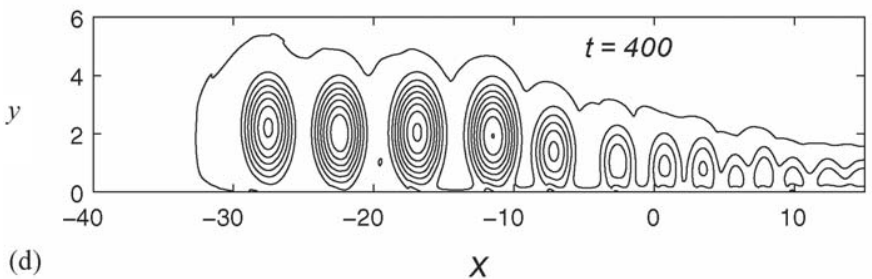

Fig. 11. The numerical solution for the geostrophically adjusted initial flow with $w_{\infty}=2$ and $L=30$. Contours of $h(x, y$, $t$ ) are shown at several times. The contour interval is 0.05. (a) $t=0$; (b) $t=20$; (c) $t=100$; (d) $t=400$.

panels the semigeostrophic solution (dashed line) is in very good agreement with the numerical solution computed with the geostrophically adjusted initial condition. Inertial oscillations excited by the release of the stagnant layer are clearly evident in the plot of $h_{\mathrm{w}}(t)$ at $x=-22.5$ in Fig. 13a. However, the oscillations are essentially superimposed on a slower adjustment that is very close to both the other numerical solution and the theoretical solution. This is also the case in Fig. 13b and c. Note that the large departures of $h_{\mathrm{w}}$ in the uniform region of Fig. 13b are due to the phase of the inertial oscillation at the particular times shown.

The evolution of the domain integrated energy,

$$
E=\iint \frac{1}{2}\left(h\left(u^{2}+v^{2}\right)+h^{2}\right) \mathrm{d} x \mathrm{~d} y,
$$

for these two model runs is shown in Fig. 14. The kinetic $\left(\iint h\left(u^{2}+v^{2}\right) / 2 \mathrm{~d} x \mathrm{~d} y\right)$ and potential $\left(\iint h^{2} / 2 \mathrm{~d} x \mathrm{~d} y\right)$ energy components are also shown. Note that only a portion of the model domain is shown in Figs. 11 and 12; however, the head of the gravity current is in the numerical domain at the times in Fig. 14. Also shown in the figure is the energy of the semigeostrophic solution 


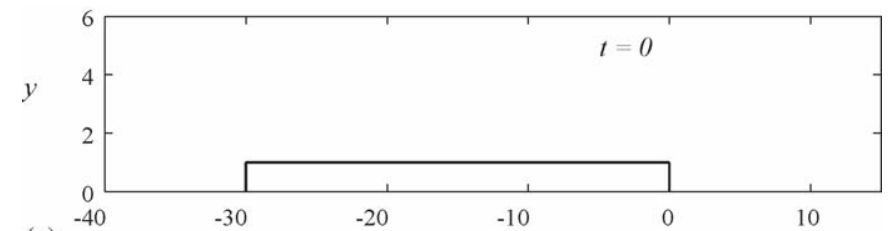

(a)

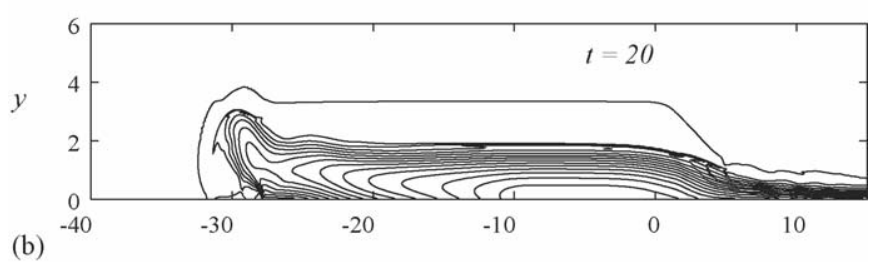

(b)

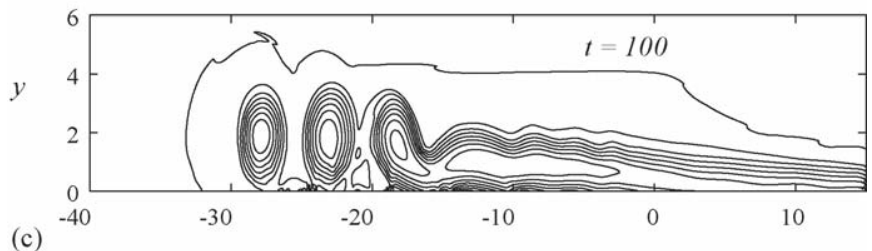

(c)

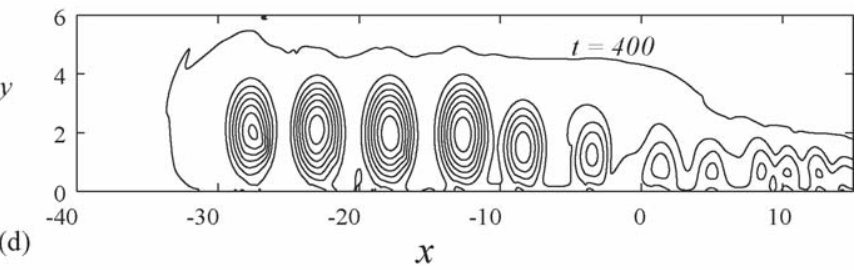

Fig. 12. The numerical solution with the stagnant layer initial condition with $w_{0}=1.03$ and $L=30$. Contours of $h(x, y$, $t$ ) are shown at several times. The contour interval is 0.05. (a) $t=0$; (b) $t=20$; (c) $t=100$; (d) $t=400$.

for $t<L / c_{\mathrm{K}} \approx 31.1$. The numerical solution with the geostrophically adjusted initial condition experiences a slow energy decay due to the breaking at the bore head. The energy decay is somewhat faster than in semigeostrophic solution, but otherwise the behavior is similar. With the stagnant pool initial condition, the energy undergoes a period of rapid decay, $0<t<2$, associated primarily with the sharp front that propagates in the offshore $(y>0)$ direction along the entire length $-L<x<0$. The breaking and rapid energy dissipation occur several more times at the inertial period. For $t>15$ the energy decays slowly and is associated with the continual dissipation at the gravity current head. The inertial oscillations continue beyond the initial rapid dissipation phase as is clear in the exchange between the kinetic and potential energies. At longer times the total energy in the run with the inertia-gravity waves is less than the initially geostrophically adjusted case. This is an irreversible effect of the early wave breaking. However, the energy difference is relatively small, less than $6 \%$ at $t=35$, and does not play a substantial role in the evolution of the flows for longer times. These results are typical of other values of $w_{0}\left(w_{\infty}\right)$.

The long-time evolution of the flow is shown in Figs. 11c and d, and $12 \mathrm{c}$ and $\mathrm{d}$ for the still initial condition. In both cases the flow rolls up into a train of anticyclonic vortices. The vortices first emerge at the upstream end, and then for increasing $x$ as $t$ increases. At $t=400$, in $-30 \leq x \leq-10$, there are four nearly identical vortices. Further downstream, the vortex radius decreases nearly 

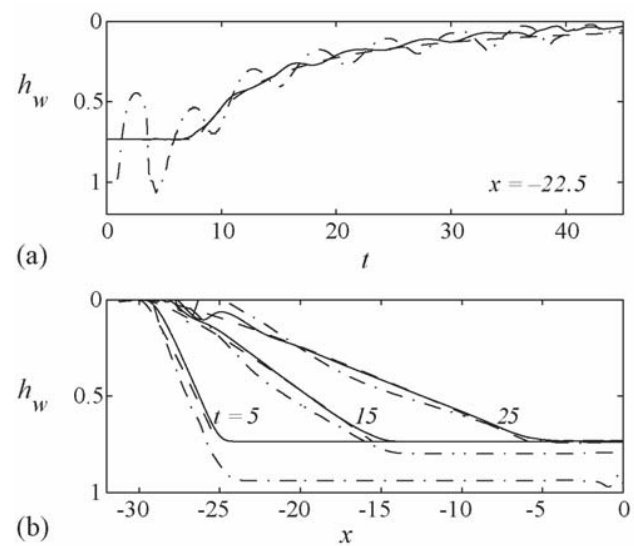

(b)

$x$

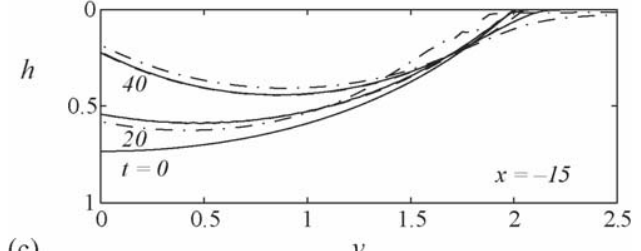

(c)

$y$

Fig. 13. Comparison of the upstream rarefaction from semigeostrophic solution (dashed) with the numerical solutions from Figs. 11(solid) and 12(dash-dot): (a) $h_{\mathrm{W}}$ vs. time at $x=-22.5$, (b) $h_{\mathrm{W}}$ at the indicated times and (c) $h(y)$ at $x=-15$ at the indicated times.

uniformly. Once formed, the large upstream vortices are stationary. They have shallow depths and weak velocities near the wall. As a consequence, image vortices required to satisfy the no normal flow condition are correspondingly weak, and thus do not advect the primary vortices. Runs with different $L(\gg 1)$ and $w_{0}$ (or $w_{\infty}$ ) give similar results, though the number of large vortices decreases with $L$ (Fig. 15).

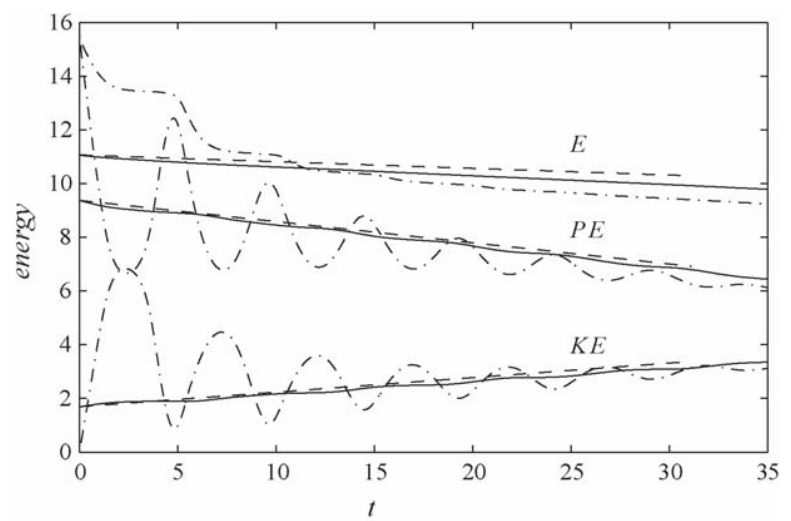

Fig. 14. The kinetic energy $\mathrm{KE}$, potential energy $\mathrm{PE}$ and total energy $E=\mathrm{KE}+\mathrm{PE}$ for the numerical runs in Figs. 11(solid) and 12(dash-dot). At the times shown, the leading edge of the gravity current is still within the model domain. The energies calculated from the semigeostrophic solution (dashed) are shown for $t<L / c_{\mathrm{K}} \approx 31.1$. 

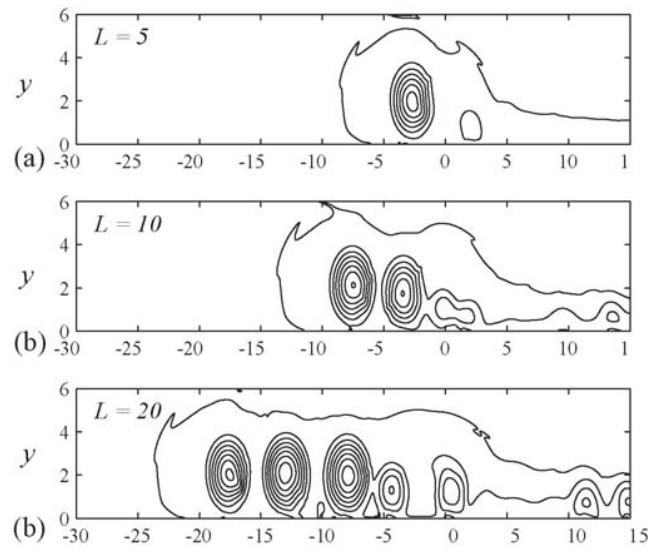

Fig. 15. Numerical solutions $h(x, y, t)$ with the still initial layer $w_{0}=1$ at $t=400$ : (a) $L=5$, (b) $L=10$ and (c) $L=20$. The contour interval is 0.05 .

A possible explanation for the vortex formation is that it results from the inertial turning of the flow at the upstream end. As each vortex pinches off, a new vortex is initiated by the inertial turning of the flow just downstream. However, if that were the case, the vortex radius should scale with the (dimensional) inertial radius $u / f$, where $u$ is some measure of the velocity. A choice for $u$ is the value at $y=0$ from (11) with $h_{\mathrm{w}}=0$ and $w=w_{\infty}$. However, the large upstream vortex radii do not scale systematically with this inertial radius.

The vortex roll-up can, however, be interpreted as a consequence of an instability of the new flow established by the Kelvin wave. The Kelvin wave passage causes the layer depth along the wall to approach zero for large time. If this new flow, with the layer depth vanishing at each edge, were uniform in $x$, it would be identical to a coupled front flow considered by Griffiths et al. (1982) and given by (10) and (11) with $h_{\mathrm{w}}=0$ and $w(x, t)$ constant. They showed that this parallel flow was linearly unstable to a mixed baroclinic-barotropic ageostrophic instability. The most unstable wavenumber $k_{\max }$ from their linear calculations is shown as a function of the current half-width $L_{1 / 2}(=w / 2)$ in Fig. 16. Also shown in the figure is the "vortex wavenumber" $2 \pi / \lambda_{\mathrm{V}}$, where $\lambda_{\mathrm{V}}$ is the center-to-center spacing of the largest upstream vortices in $-L \leq x \leq 0$. These

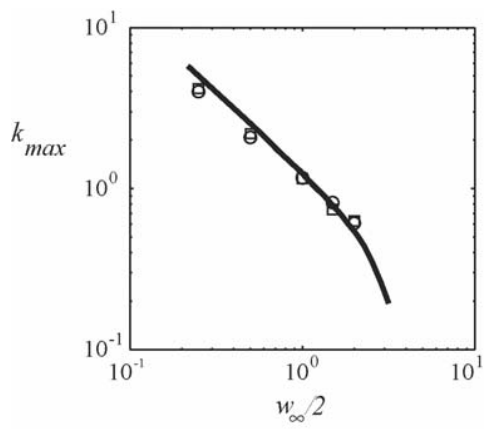

Fig. 16. The wavenumber of maximum linear growthrate $k_{\max }$ vs. the current half-width $L_{1 / 2}=w_{\infty} / 2$ from Griffiths et al. (1982) is shown by the line. The circles show the vortex wavenumber, $2 \pi / \lambda_{\mathrm{V}}$, from the numerical solutions of the adjustment problem. The vortex wavenumbers from numerical solutions of the coupled front parallel flow are indicated by the squares. 


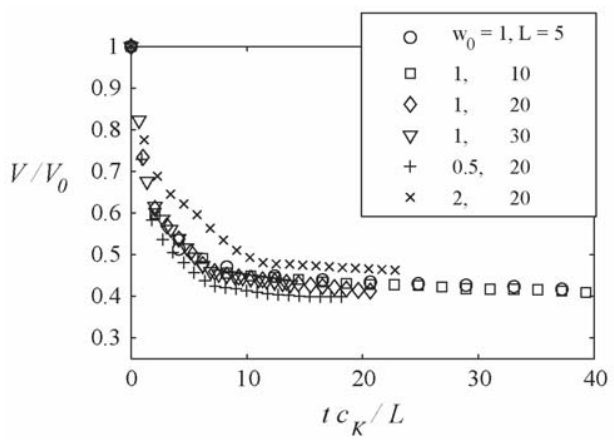

Fig. 17. The fraction of fluid in $x<0, V / V_{0}$, vs. scaled time $t c_{\mathrm{K}} / L$.

values are plotted versus $w_{\infty} / 2$, the approximate half-width of the flow established by the Kelvin wave propagation, and were found from runs with $L$ large enough to give at least four large nearly identical vortices. The vortex wavelength is given quite well by the wavelength of the fastest growing linear wave from the Griffiths et al. (1982) theory.

As a further test of the connection between these results and the Griffiths et al. (1982) instability, a set of numerical calculations was conducted to explore the finite-amplitude development of the instability in a parallel flow well removed from the boundary. The initial condition was given by given by (10) and (11) with $h_{\mathrm{w}}=0$ and $w(x, t)=w_{\infty}$ plus small random fluctuations in $x$. The domain was periodic in $x$ with length 60 and extended in $y$ so that the current never encountered a boundary. Runs with $w_{\infty}=0.5-4$ were made. In all cases the flow evolved to a train of nearly identical, stationary anticyclonic vortices. The vortex wavenumbers from these runs are also shown in Fig. 16 and are very close to the results from the localized adjustment problem. While the flow established by the Kelvin wave in the adjustment problem is neither steady nor uniform in $x$ as required by the linear theory, it seems reasonable to interpret the development of the vortices as the finite-amplitude manifestation of the linear instability found by Griffiths et al. (1982).

Finally, the development of the stationary vortex array leads to the trapping of some of the initial volume, $V_{0}=w_{0} L$, in the neighborhood of the initial layer. For example, in Fig. 11d just under $42 \%$ of $V_{0}$ remains in $x<0$ at $t=400$. Fig. 17 shows the fluid volume in $x<0, V=\int_{-\infty}^{0} \int_{0}^{\infty} h \mathrm{~d} y \mathrm{~d} x$, as a function of time for a number of runs with $w_{0}=0.5-2$ and $L=5-30$. When $t$ is scaled by $L / c_{\mathrm{K}}$, the time for the Kelvin wave to reach the downstream end of the initial layer, and $V$ by $V_{0}$, the data collapse reasonably well. About $40-45 \%$ of the fluid remains in $x<0$ and occurs by $t \approx 10 L / c_{\mathrm{K}}$. The trapped mass fraction increases slightly with $w_{0}$, but is essentially independent of $L$.

\section{Conclusion}

The semigeostrophic theory and numerical calculations have explored the nonlinear, longtime evolution of a finite layer of buoyant, uniform potential vorticity fluid released adjacent to a wall in a rotating system. Far from the two ends of the layer the interior flow is initially given by the classic one-dimensional geostrophic adjustment solution. The main results address the additional processes associated with adjustment at the upstream and downstream ends of the layer and touch on the role of inertia-gravity waves excited in the initial adjustment. The resulting flow produces a gravity current at the downstream end and a Kelvin rarefaction 
from the upstream end. The latter modifies the interior geostrophic flow, setting the conditions for an instability that ultimately leads to a train of stationary, anticyclonic vortices along the boundary.

The inertia-gravity waves excited by the adjustment are highly nonlinear and break, but do not significantly affect the slower geostrophic flow. This somewhat surprising result may, though, be a consequence of the uniform potential vorticity of the initial condition. The strong anisotropy inherent in the initial condition and resulting flow may also be a factor (cf. Hayasha and Young, 1987). It would also be interesting to consider initial conditions that promote strong gradients in wave breaking which then give rise to potential vorticity changes and thus a more direct and faster interaction.

Despite the simplifications inherent in the reduced-gravity model, the results here should provide some guidance when considering the evolution of similar flows in the ocean and atmosphere. There are, however, several aspects of the model that perhaps restrict direct application. The first is the limitation to one active layer. In the coastal ocean the lower layer can rarely be considered dynamically inactive. An active lower layer will not eliminate the generation of a gravity current, but may change its properties. In principle it should be possible to include an active lower layer in the semigeostrophic theory, though this would greatly complicate the analysis. In addition to following the evolution of the upper layer front defining the gravity current, the model would need to include the motion of a potential vorticity front in the lower layer. The upstream Kelvin rarefaction should also proceed essentially as discussed here. However, any instability, and subsequent finite-amplitude development, of the flow will be modified by the presence of the lower layer (Paldor and Ghil, 1990; Reszka and Swaters, 1999). For example, upper layer anticyclones could couple with lower layer cyclones and propagate away from the wall. Indeed, some preliminary numerical calculations with a two-layer version of the model indicate that this is the case.

One might also wonder if friction, specifically no-slip boundaries, alters the results. The question of lateral friction on rotating gravity currents was considered in Helfrich and Mullarney (2005) where it was shown that a no-slip wall causes a slow decay of the gravity current speed and a tapering, rather than uniform width gravity current. The effect of a no-slip condition on the upstream flow can be anticipated to be weak, provided that the Reynolds number is not too large. This is because the geostrophic flow (2) already satisfies the no-slip condition. The upstream Kelvin rarefaction could be affected. But a numerical test (not shown) with Laplacian friction with a Reynolds number $\sqrt{g^{\prime} H} L_{\mathrm{R}} / v=10^{3}$ still produced a train of standing vortices, though they were weaker than the equivalent inviscid case.

Perhaps the most important aspect of any realistic flow absent in this reduced-gravity model is topography. Topography could enter as a bottom sloping away from a vertical wall. In that case, if the upper layer did not contact the bottom, the downstream gravity current evolution would probably be unaffected by the bottom slope, though any instability of the upstream flow would be (Reszka and Swaters, 1999). Of even more relevance is a sloping bottom that intersects the free surface, eliminating the vertical boundary. Then nearly all aspects of the evolution would be modified, though the qualitative aspects would likely remain. The interior geostrophic adjustment would depend on the slope (Hsueh and Cushman-Roisin, 1983) and be fundamentally influenced by bottom Ekman processes (Chapman and Lentz, 1994). The downstream flow would evolve into a gravity current, but as shown by Lentz and Helfrich (2002), the gravity current response can vary from the Kelvin wave-like gravity current regime against a vertical wall to a slower and wider topographic wave controlled gravity current for small bottom slopes. These roles of an active lower layer and a bottom slope are the subjects of ongoing work. 


\section{Acknowledgment}

This work was supported by NSF Grant OCE-0325102.

\section{References}

Abbott, M.B., 1961. On the spreading of one fluid over another. Part ii. The wave front. La Houille Blanche 6, 827-846.

Benjamin, T.B., 1968. Gravity currents and related phenomena. J. Fluid Mech. 31, 209-248.

Blumen, W., 1972. Geostrophic adjustment. Rev. Geophys. Space Phys. 10, 485-528.

Chapman, D.C., Gawarkiewicz, G., 1997. Shallow convection and buoyancy equilibration in an idealized coastal polynya. J. Phys. Oceanogr. 27, 555-566.

Chapman, D.C., Lentz, S.J., 1994. Trapping of a coastal density front by the bottom boundary layer. J. Phys. Oceanogr. 24, 1464-1479.

Dorman, C.E., 1987. Possible role of gravity currents in northern California's coastal summer wind reversals. J. Geophys. Res. 92, 1497-1506.

Fedorov, A., Melville, W., 1996. Hydraulic jumps at boundaries in rotating fluids. J. Fluid Mech. 324, 55-82.

Gill, A.E., 1976. Adjustment under gravity in a rotating channel. J. Fluid Mech. 77, 603-621.

Griffiths, R.W., Hopfinger, E.J., 1983. Gravity currents moving along a lateral boundary in a rotating fluid. J. Fluid Mech. 134, 357-399.

Griffiths, R.W., Killworth, P.D., Stern, M.E., 1982. Ageostrophic instability of ocean currents. J. Fluid Mech. 117, 343-377.

Hacker, J.N., Linden, P.F., 2002. Gravity currents in rotating channels. Part 1. Steady-state theory. J. Fluid Mech. 457, 295-324.

Helfrich, K.R., Kuo, A.C., Pratt, L.J., 1999. Nonlinear Rossby adjustment in a channel. J. Fluid Mech. 390, $187-222$.

Helfrich, K.R., Mullarney, J., 2005. Gravity currents from a dam-break in a rotating channel. J. Fluid Mech. 536, $253-283$.

Hermann, A.J., Rhines, P.B., Johnson, E.R., 1989. Nonlinear Rossby adjustment in a channel: beyond Kelvin waves. J. Fluid Mech. 205, 469-502.

Hayasha, Y.-Y., Young, W.R., 1987. Stable and unstable shear modes of rotating parallel flow in shallow water. J. Fluid Mech. 184, 477-504.

Hsueh, Y., Cushman-Roisin, B., 1983. On the formation of surface to bottom fronts over steep topography. J. Geophys. Res. 88, 743-750.

Huppert, H., Simpson, J.E., 1980. The slumping of gravity currents. J. Fluid Mech. 99, 785-799.

Killworth, P.D., 1992. The time-dependent collapse of a rotating fluid cylinder. J. Phys. Oceanogr. 22, 390-397.

Klemp, J.B., Rotunno, R., Skamrock, W.K., 1994. On the dynamics of gravity currents in a channel. J. Fluid Mech. 269, 169-198.

Klemp, J.B., Rotunno, R., Skamrock, W.K., 1997. On the propagation of internal bores. J. Fluid Mech. 331, 81-106.

Kubokawa, A., Hanawa, K., 1984. A theory of semigeostrophic gravity waves and its application to the intrusion of a density current along a coast. Part 2 . Intrusion of a density current along a coast of a rotating fluid. J. Oceanogr. Soc. Jpn. 40, 260-270.

Kuo, A.C., Polvani, L.M., 1997. Time-dependent fully nonlinear geostrophic adjustment. J. Phys. Oceanogr. 27, 1614-1634.

Lentz, S.J., Helfrich, K.R., 2002. Buoyant gravity currents along a sloping bottom in a rotating channel. J. Fluid Mech. 464, 251-278.

Leveque, R.J., 2002. Finite Volume Methods for Hyperbolic Problems. Cambridge University Press.

Paldor, N., Ghil, M., 1990. Finite-wavelength instabilities of a coupled density front. J. Phys. Oceanogr. 20, $114-123$.

Pratt, L.J., 1983. On inertial flow over topography. Part 1. Semigeostrophic adjustment to an obstacle. J. Fluid Mech. 131, 195-218.

Pratt, L.J., Helfrich, K.R., Chassignet, E.P., 2000. Hydraulic adjustment to an obstacle in a rotating channel. J. Fluid Mech. 404, 117-149.

Reszka, M.G., Swaters, G.E., 1999. Eddy formation and interaction in a baroclinic frontal geostrophic model. J. Phys. Oceanogr. 29, 3025-3042.

Reznik, G.M., Zeitlin, V., Ben Jelloul, M., 2001. Nonlinear theory of geostrophic adjustment. Part 1. Rotating shallowwater model. J. Fluid Mech. 445, 93-120.

Reznik, G.M., Grimshaw, R., 2002. Nonlinear geostrophic adjustment in the presence of a boundary. J. Fluid Mech. 471, 257-283. 
Rossby, C.G., 1938. On the mutual adjustment of pressure and velocity distributions in certain simple current systems ii. J. Mar. Res. 1, 239-263.

Stern, M.E., 1980. Geostrophic fronts, bores, breaking and blocking waves. J. Fluid Mech. 99, 687-703.

Stern, M.E., Helfrich, K.R., 2002. Propagation of a finite-amplitude potential vorticity front along the wall of a stratified fluid. J. Fluid Mech. 468, 179-204.

Stern, M.E., Whitehead, J.A., Hua, B.L., 1982. The intrusion of a density current along the coast of a rotating fluid. J. Fluid Mech. 123, 237-265.

Stoker, J.J., 1957. Water Waves. Interscience.

Tommason, G.G., Melville, W.K., 1992. Geostrophic adjustment in a channel: nonlinear and dispersive effects. J. Fluid Mech. 241, 23-58.

Whitham, G.B., 1974. Linear and Nonlinear Waves. Wiley. 